\title{
Non-echoplanar diffusion weighted imaging in the detection of post-operative middle ear cholesteatoma: navigating beyond the pitfalls to find the pearl
}

\author{
Ravi K. Lingam ${ }^{1} \cdot$ Robert Nash $^{2} \cdot$ Anooj Majithia ${ }^{2} \cdot$ Ali Kalan $^{2} \cdot$ Arvind Singh $^{2}$
}

Received: 18 May 2016 /Revised: 27 July 2016 / Accepted: 4 August 2016 / Published online: 24 August 2016

(C) The Author(s) 2016. This article is published with open access at Springerlink.com

\begin{abstract}
Non-echoplanar diffusion weighted magnetic resonance imaging (DWI) has established itself as the modality of choice in detecting and localising post-operative middle ear cleft cholesteatoma. Despite its good diagnostic performance, there are recognised pitfalls in its radiological interpretation which both the radiologist and otologist should be aware of. Our article highlights the various pitfalls and provides guidance for improving radiological interpretation and navigating beyond many of the pitfalls. It is recommended radiological practice to interpret the diffusion weighted images together with the ADC map and supplement with the corresponding $\mathrm{T} 1$ weighted and T2 weighted images, all of which can contribute to and enhance lesion localisation and characterisation. ADC values are also helpful in improving specificity and confidence levels. Given the limitation in sensitivity in detecting small cholesteatoma less than $3 \mathrm{~mm}$, serial monitoring with DWI over time is recommended to allow any small residual cholesteatoma pearls to grow and become large enough to be detected on DWI. Optimising image acquisition and discussing at a joint clinico-radiological meeting both foster
\end{abstract}

This paper was presented as a scientific oral presentation at the European Society of Head \& Neck Radiologists (ESHNR) annual scientific meeting 2015 in Krakow.

Ravi K. Lingam

ravi.lingam@nhs.net

1 Department of Radiology, Northwick Park and Central Middlesex Hospitals, London Northwest Healthcare NHS Trust, London, England, UK

2 Department of Otolaryngology, Head \& Neck Surgery, Northwick Park and Central Middlesex Hospitals, London Northwest Healthcare NHS Trust, London, England, UK good radiological interpretation to navigate beyond the pitfalls and ultimately good patient care.

Teaching Points

- Non-echoplanar DWI is the imaging of choice in detecting post-operative cholesteatoma.

- There are recognised pitfalls which may hinder accurate radiological interpretation.

- Interpret with the ADC map /values and T1W and T2W images.

- Serial DWI monitoring is of value in detection and characterisation.

- Optimising image acquisition and discussing at clinicoradiological meetings enhance radiological interpretation.

Keywords Diffusion weighted imaging $\cdot$ MRI ·

Cholesteatoma $\cdot$ Pitfalls $\cdot$ DWI

\section{Introduction}

Since it was first described in 2006 for the detection of cholesteatoma [1,2], non-echoplanar diffusion weighted magnetic resonance imaging (DWI) has now firmly established its role as the imaging modality of choice in detecting post-operative cholesteatoma [3-7]. It has superseded CT, echoplanar DWI and delayed contrast MRI by virtue of its superior diagnostic performance [3-8]. Nonechoplanar DWI is capable of acquiring thin slices (as thin as $2 \mathrm{~mm}$ ) and generating a high resolution matrix, and hence can detect cholesteatoma as small as $2 \mathrm{~mm}$ [3-5]. It also has the advantage of not requiring intravenous gadolinium contrast, which is implicated with nephrogenic systemic fibrosis [9] and intracerebral deposition [10]. It also does not require delayed scans as with 
delayed contrast MRI [3]. Even though echoplanar DWI has a shorter acquisition time, non-echoplanar DWI performs better than its echoplanar counterpart primarily due to the lack of air-bone susceptibility artefact and distortion at the temporal bones $[3,6]$. It offers the potential to reduce the number of mandatory second-look (or relook) surgery in detecting residual or recurrent disease $[3,5,7]$. However, the limitations of the technique still need to be considered as part of a safer and cost-effective work-up strategy. This article aims to highlight pitfalls of radiological interpretation and empower the reader with necessary approaches to navigate beyond the pitfalls.

\section{Optimisation of technique}

By virtue of its keratin content, cholesteatoma returns high signal intensity compared to brain tissue on the diffusion weighted images obtained both at $b$ values of $0 \mathrm{~s} / \mathrm{mm}^{2}$ and at higher values of 800 or $1000 \mathrm{~s} / \mathrm{mm}^{2}$ (Fig. 1). This is due to a combination of restricted diffusion and $\mathrm{T} 2$ shine-through effect. It returns low signal on the apparent diffusion coefficient (ADC) map $[3,4,9]$. The ADC map is free from any T1 or T2 effects, provides a true quantitative display of water diffusivity at each voxel [11], and is integral in the interpretation of the DWI images [3, 12]. Non-cholesteatomatous soft tissue such as granulation tissue, inflammation, and fluid return lower or no signal on the high $b$ value (b800 or b1000) images compared to the $\mathrm{b} 0$ images and consequently high signal on the ADC map (Fig. 2).

Fig. 1 Typical MRI

characteristics of post-operative cholesteatoma. Coronal images through the left mastoid remnant shows the lesion (white arrow) as high signal on a the $\mathrm{T} 2$ weighted image, b DWI b0 image, and c DWI b1000 image and a low signal on the $\mathbf{d}$ ADC map and $\mathbf{e}$ non-contrast enhanced $\mathrm{T} 1$ weighted image. f Surgery confirms the presence of the cholesteatoma. The keratin has been suctioned away showing the underlying white squamous epithelium lining the mastoid remnant (white arrow) and aditus (star)
To allow accurate radiological interpretation, the DWI images need to be optimal in the first instance. The nonechoplanar technique can generate image slices without airbone susceptibility artefacts and distortion, as thin as $2-3 \mathrm{~mm}$ producing a high resolution matrix, which is essential for the detection of tiny cholesteatoma pearls (Fig. 3). Current nonechoplanar sequences broadly include single-shot turbo spinecho (SSTSE) sequences [such as HASTE (half Fourier SSTSE) [13-19]] and multishot turbo spin-echo (MSTSE) sequences [such as with PROPELLER (periodically rotated overlapping parallel lines with enhanced reconstruction) [20-23]]. Currently, slice thickness as low as $2 \mathrm{~mm}$ can be acquired with HASTE and $3 \mathrm{~mm}$ with PROPELLER DWI [4]. The SSTSE sequence such as HASTE acquire one $b$ value at a time (currently 5 to $6 \mathrm{~min}$ for each b value) and are susceptible to both movement artefacts and slice position misregistration errors in post-acquisition ADC map calculation. In contrast, the multidirectionally acquired sequences, however, can acquire two $b$ values in a single acquisition (currently under 5 min with multishot PROPELLER DWI), and hence theoretically quicker and less susceptible to motion artefacts and slice position misregistration errors than HASTE [4]. The MSTSE sequences have a shorter echo train than their single shot counterparts and hence are able to mitigate the T2 blurring effects (image degradation due to $\mathrm{T} 2$ decay during acquisition) [24]. As a SSTSE sequence, but using a half-Fourier acquisition, HASTE is also able to reduce the effect of T2 blurring. With most types, non-echoplanar diffusion weighted images can be acquired in any plane. Currently, with the PROPELLER DWI sequence, the images can currently only
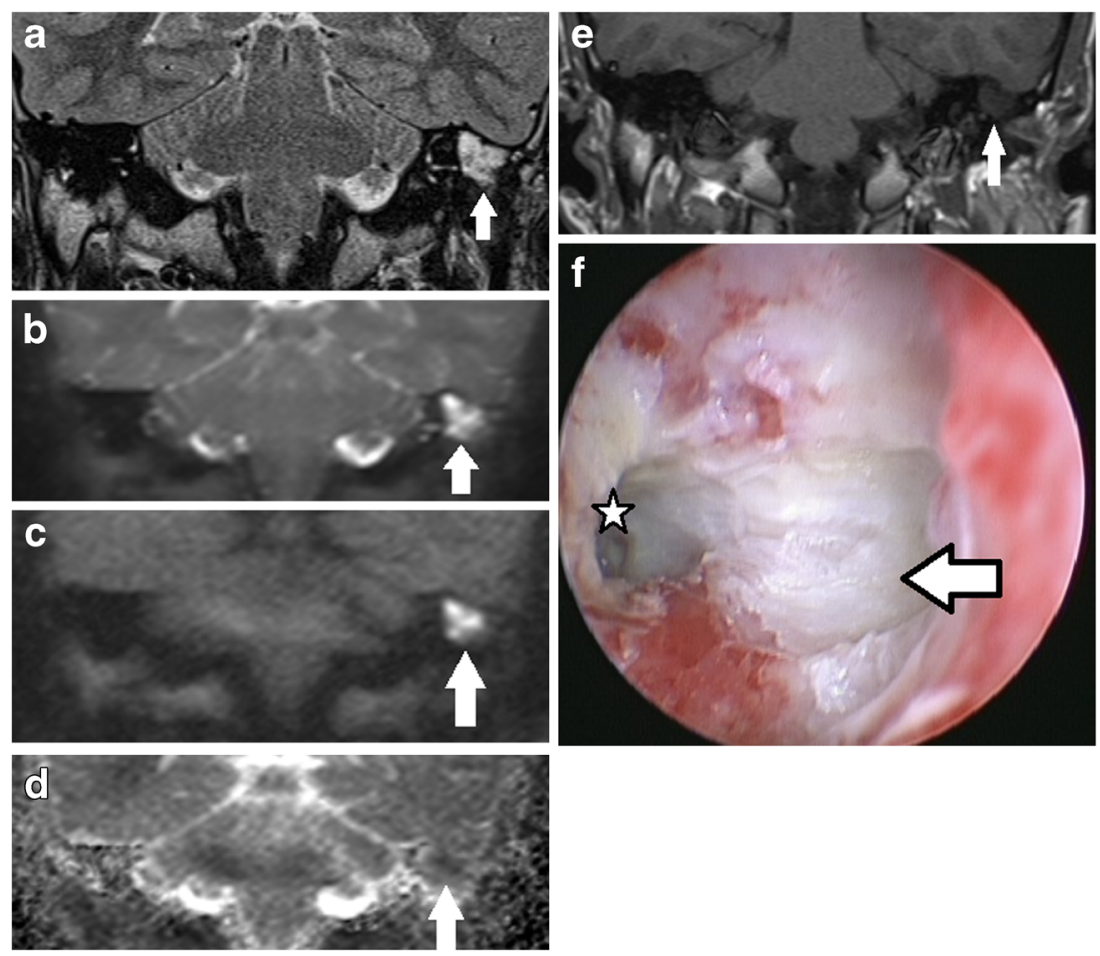

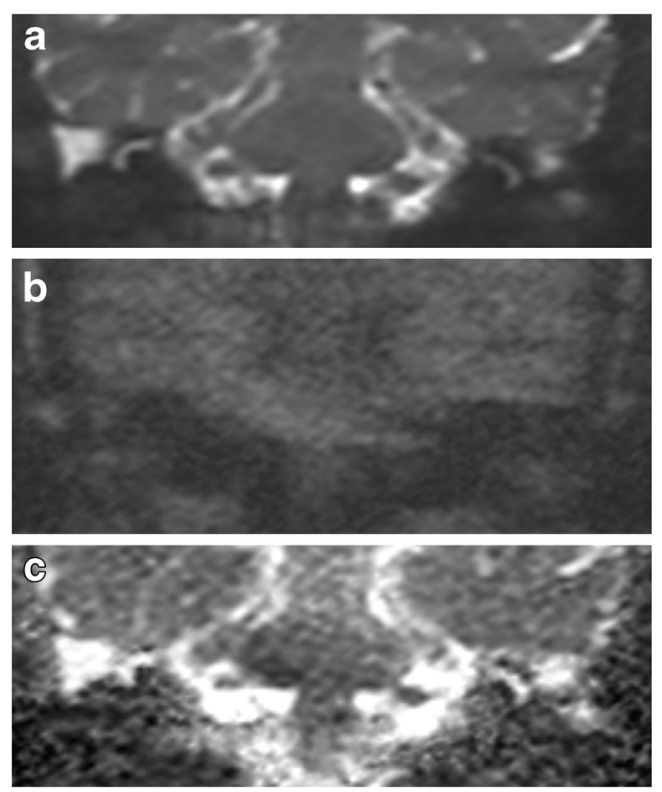

Fig. 2 Typical DWI appearance of non-cholesteatomatous soft tissue. Coronal images through the right post-operative mastoid remnant shows the soft tissue (white arrow) as high signal on a the b0 image, but loses signal on b the b1000 image, and hence demonstrates high signal on $\mathbf{c}$ the ADC map. Surgery confirms granulation tissue

be acquired in the axial plane [3, 4, 20,23], but it can be reconstructed in other planes [21]. Three dimensional (3D) DWI acquisition with multiplanar reconstruction has also been described with TFE-DSDE [Turbo field-echo with diffusion-sensitized driven equilibrium] [25]. In our institution, the coronal images are preferred as it demonstrates the tegmen and the relevant tympanomastoid anatomy better for more confident localisation of disease and surgical planning. Ideally, the DWI sequences should be supplemented by conventional $\mathrm{T} 2$ weighted and $\mathrm{T} 1$ weighted images of similar slice thickness and slice positions to the DWI images to obtain the anatomical information required for better localisation of disease. On conventional MRI, cholesteatoma typically returns high signal on the T2 weighted MR images and low signal on the T1 weighted MR images (Fig. 1) [3]. Though not specific, these sequences, together with the DWI sequences, can help further characterise the disease and detect cholesteatoma more accurately [4]. Supplementing with post-gadolinium enhanced contrast scan is not necessary as it does not confer greater diagnostic performance than nonechoplanar DWI alone [8].

Typically, two $b$ values are sufficient to generate the ADC map, which is reconstructed following acquisition of the scans [12]. More $b$ values can be used to strengthen further the ADC map, but would incur a longer scanning time and a higher risk of patient movement during scanning and image misregistration. Crucially, for non-multidirectionally acquired sequences (such as HASTE), which require separate acquisitions for each $b$ value, the measurement parameters and the slice positions for the various b values have to be identical (copyreferenced) to promote better image registration and generation of the ADC map. Getting the patient to co-operate by keeping still during DWI scanning is vital for achieving good slice registration and image quality.

\section{Quantitative assessment with ADC values}

Cholesteatomas have been shown to have a significantly lower ADC value than non-cholesteatomatous soft tissue [12]. The threshold ADC values for differentiating the tissue types need to take into account data acquisition parameters $[12,26]$. Once the ADC threshold is established for the scanner and the scanning protocol, it performs well in discriminating cholesteatoma from non-cholesteatomatous
Fig. 3 DWI appearance of a small residual cholesteatoma confirmed on second-look surgery. The $\mathbf{a}$ b0 and $\mathbf{b}$ b 1000 image shows a small $5 \mathrm{~mm}$ high signal cholesteatoma (white arrow) at the right epitympanum. It returns low signal on $\mathbf{c}$ the corresponding ADC map, high signal on $\mathbf{d}$ the $\mathrm{T} 2$ weighted image and low signal on $\mathbf{e}$ the non-contrast enhanced T1 weighted image
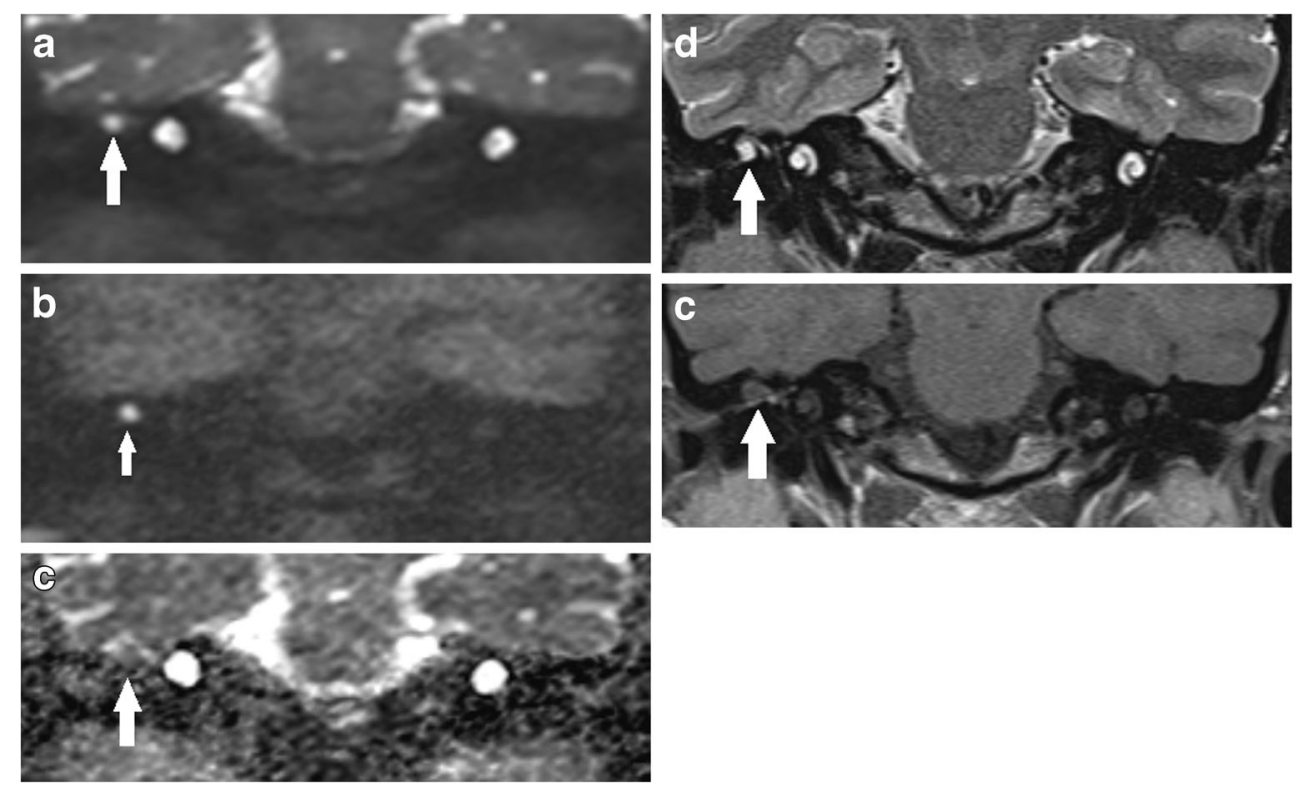

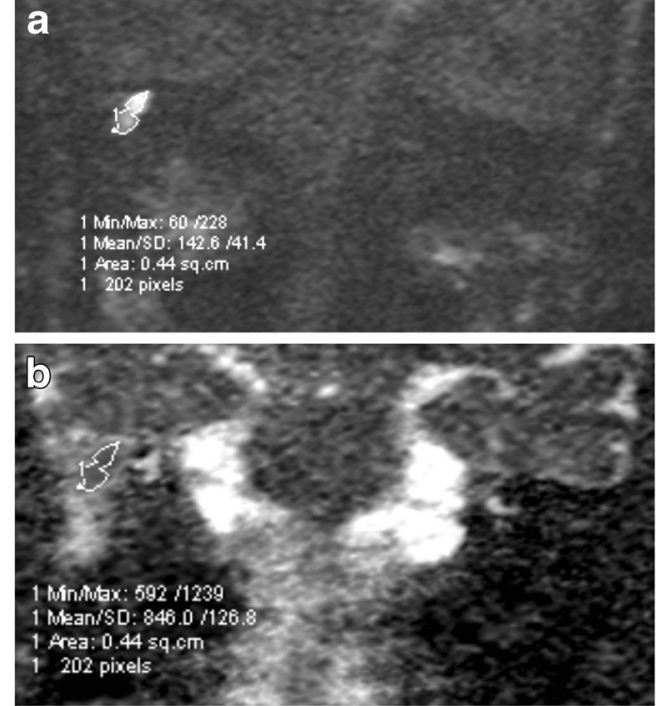

Fig. 4 Measuring the ADC value of a lesion. a As the lesion was better seen on the b1000 image, a freehand region of interest (ROI) was contoured around the inner border of the lesion. An image was selected so the central part of the lesion was measured to avoid partial volume averaging. The software then maps the ROI on $\mathbf{b}$ the corresponding ADC map where the $A D C$ value is automatically calculated $A D C$ value $846 \times 10^{-6} \mathrm{~mm}^{2} / \mathrm{s}$. For accuracy, this can be repeated several times and a median reading obtained. In some cases, if the lesion is well seen on the ADC map, the ROI can be placed directly on the map for ADC value calculation

soft tissue with comparable sensitivity and marginally higher specificity than the qualitative method and can help navigate beyond some radiological interpretation pitfalls [12]. The ADC value can be measured by simply placing on the ADC map a region of interest (ROI) on the lesion, preferably on an image section that demonstrates the lesion with the highest contrast. For a more accurate measurement, a freehand ROI can be used and contoured around the inner border of the central aspect of the lesion to avoid the edges and partial volume averaging, and a median of several ADC values is obtained [12, 26] (Fig. 4). When residual lesions are small and seen only on two contiguous sections, the mean ADC value can be taken from the two sections for a more representative value $[12,26]$.

\section{Diagnostic performance in detecting post-operative cholesteatoma}

Following canal wall up mastoidectomy, a mandatory secondlook surgery is performed 9-12 months later to detect residual disease. This is because the current literature suggests that canal wall up procedures are associated with rates of residual and recurrent disease of anything up to 36 and $18 \%$, respectively $[3,27,28]$.

There are many studies to date that have evaluated the performance of non-echoplanar DWI in detecting postoperative cholesteatoma $[2,8,12-23,25,29-39]$. They are all observational studies with some studies having mixed postoperative and primary cholesteatoma cases in their study samples. The studies include both prospective cohort and retrospective studies, with sample sizes up to 158 patients [38]. The vast majority of the studies demonstrate a sensitivity
Fig. 5 False negative case for cholesteatoma on DWI. a, $\mathbf{b}$ A small $2 \mathrm{~mm}$ cholesteatoma pearl (white arrow) was found on surgery in the hypotympanum (star denotes posterior canal wall), but was not depicted on $\mathbf{c}$ the corresponding $2 \mathrm{~mm}$ thick coronal b1000 image or $\mathbf{d}$ T2 weighted image
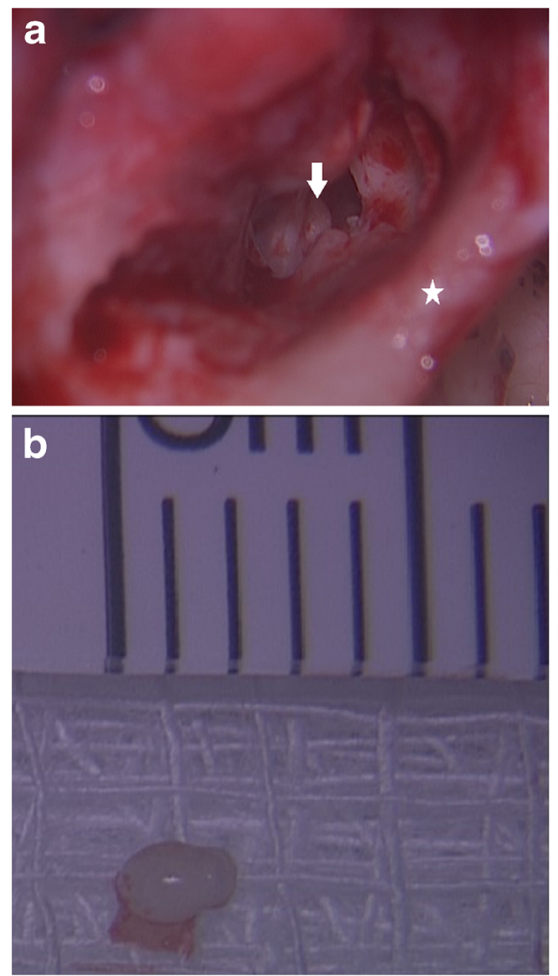
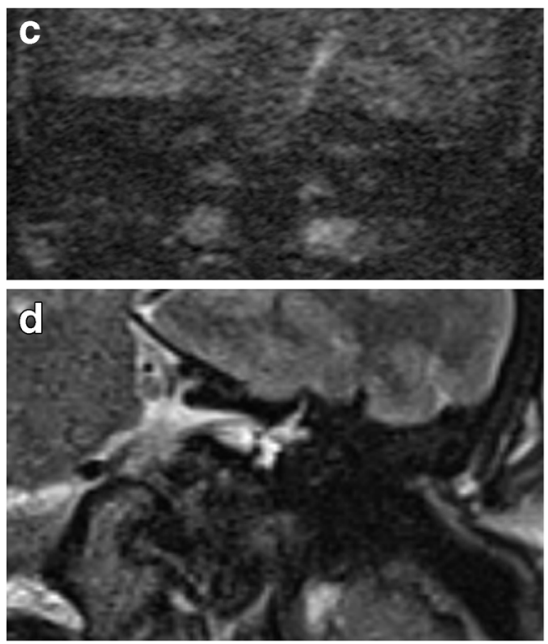

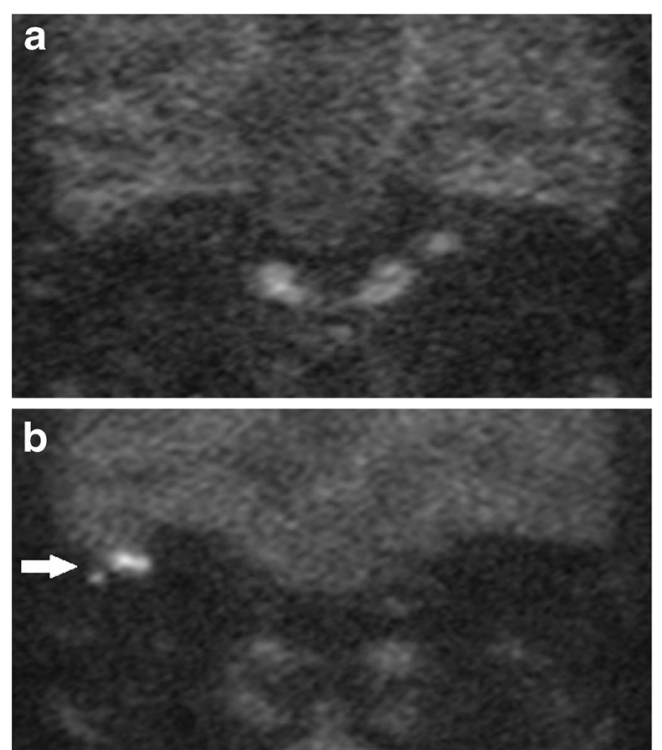

Fig. 6 Value of monitoring DWI scans which are negative for the presence of post-operative cholesteatoma. A year following right canal wall up surgery, the DWI scan was negative as shown by the a coronal b1000 image. The patient opted for monitoring with DWI instead of second-look surgery as his ear was dry. b A follow-up DWI scan a year later showed residual cholesteatoma (white arrow), which was confirmed on subsequent surgery

and specificity between 80 and $100 \%$ in detecting middle ear cholesteatoma. A previous meta-analysis of 10 studies in 2013 reported a sensitivity and specificity of $94 \%$ in detecting middle ear cholesteatoma [5].

The high diagnostic performance of DWI in detecting postoperative cholesteatoma lends support for a non-invasive alternative for reliably detecting post-operative disease. Apart from detecting post-operative cholesteatoma, DWI has also been shown to correlate well with surgery in depicting size
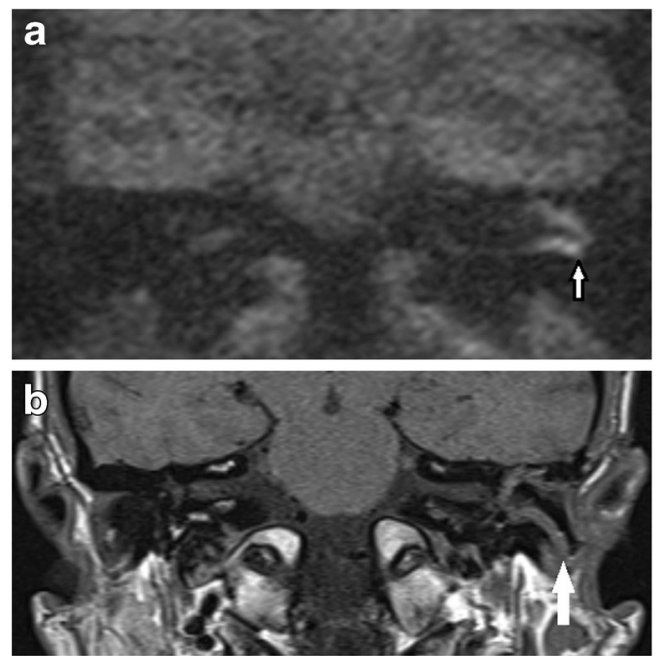

Fig. 7 Case of wax in the ear canal (white arrow) mimicking residual disease. The a b1000 image shows parallel high signal, which corresponded to wax in the adjacent ear canal, localised better on $\mathbf{b}$ the corresponding non-contrast enhanced $\mathrm{T} 1$ weighted image and location of the disease [18]. It therefore provides useful information for both operative planning and patient counselling. Despite its high diagnostic performance, the technique is associated with some pitfalls in image interpretation attributed by limitations in the technique, variability in the nature and behaviour of cholesteatoma and characteristics and complexity of the reconstructed post-operative anatomy.

\section{Pitfalls in sensitivity}

A high sensitivity is clinically more desirable than a high specificity if DWI were to replace mandatory second-look surgery as it will reduce residual or recurrent cholesteatomas from being missed. The studies have shown consistently reported that DWI is limited in its ability to detect small cholesteatoma pearls less than $3 \mathrm{~mm}$ (Fig. 5). Occasionally slightly larger cholesteatoma up to $5 \mathrm{~mm}$ can be missed, presumably due to the nature of the cholesteatoma (lack of necessary keratin to return
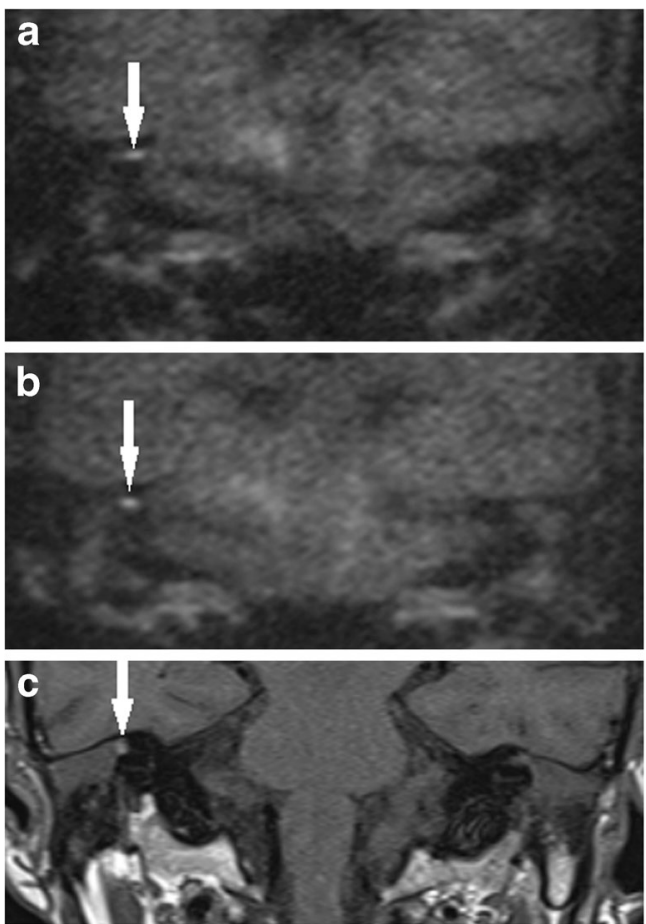

Fig. 8 False positive case of proteinaceous fluid in the mastoid remnant. The coronal a b1000 image shows a small focus of high signal (white arrow) postero-medially at the mastoid remnant, which returned low signal on the ADC map, but was distant from the site of the original middle ear cholesteatoma which was excised with high surgical confidence. At the clinico-radiological meeting, it was decided to monitor this annually with DWI. b The subsequent coronal b1000 image showed the high signal focus (white arrow) remained unchanged over 2 years making it more unlikely to represent residual disease. $\mathbf{c}$ The corresponding non-contrast enhanced $\mathrm{T} 1$ weighted image showed the lesion (white arrow) returned high $\mathrm{T} 1$ signal suggestive of proteinaceous fluid given its stability 
high DWI signal or poor image quality (movement artefact) $[3,18,38]$. There are a few studies in the literature with lower reported sensitivities than expected, this being attributed to a larger proportion in their study sample of cholesteatoma less than $3 \mathrm{~mm}$ in size [17, 29]. In addition, no difference was demonstrated between the performance using a $3 \mathrm{~T}$ scanner $[17,25]$ and a $1.5 \mathrm{~T}$ scanner, both equally limited in reliably detecting cholesteatomas less than $2-3 \mathrm{~mm}$. Other less common causes of false negative cases described in the current literature include autoatticotomy (an epithelial lined sac) [8, 23, 29, 32], aspiration of cholesteatoma on microsuction [23], and patient movement artefacts [37].

\section{Navigating past the pitfalls in sensitivity}

Avoiding a second-look or re-look surgery with a noninvasive technique such as DWI has financial benefits and the advantage of reducing surgical morbidity [3]. Despite the high diagnostic performance of DWI, the inability of a negative DWI scan to entirely exclude the presence of a small cholesteatoma impacts its potential to replace mandatory second-look surgery. When faced with a negative DWI, the decision to perform secondlook surgery depends on many factors, including current clinical/otoscopic signs, the extent of initial disease and
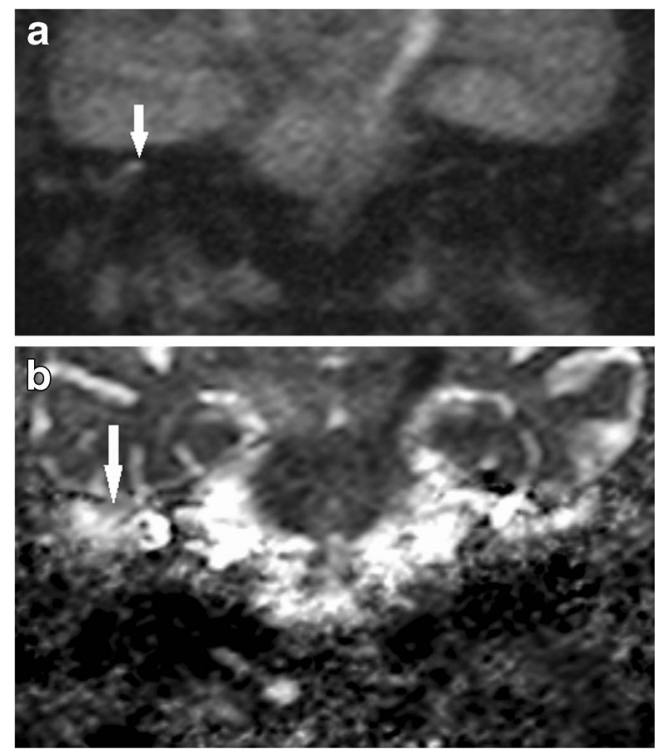

Fig. 9 False positive high DWI signal for cholesteatoma. Noncholesteatomatous tissue (surgically confirmed non-specific inflammation) at the post-operative mastoid remnant, which demonstrated a high signal (white arrow) on the b1000 image suggestive of residual cholesteatoma. However, b the corresponding ADC map demonstrated borderline high ADC signal (white arrow) and value not supporting the diagnosis of residual cholesteatoma. This case could also have been resolved with a follow-up DWI scan clinical confidence in surgical clearance at initial surgery, patient preference, and other risk factors of surgery such as patient's age and co-morbidity [3]. Several authors have proposed a reasonable approach such as replacing second-look surgery in low-risk cases and following them up with serial annual DWI over a reasonable length of time $[3,18,38]$. This will allow time for any small undetected cholesteatoma to grow and accumulate keratin to become sufficiently large to be detectable on DWI (Fig. 6). A recent study demonstrated that in patients with a negative first post-operative follow-up DWI and no clinical signs of post-operative cholesteatoma, a repeat second-follow up scan (performed at least 6 months after first follow-up DWI) was positive or equivocal for disease in 14 out of $45(31 \%)$ ears, with five ears having cholesteatoma confirmed on surgical exploration [40]. In addition, 2 third-follow-up DWI scans, which were previously negative, turned positive for cholesteatoma and was confirmed on surgery. It is, however, currently unclear what the optimal length and frequency should be for serial follow-up imaging with DWI, and this is still subject of research and discussion. The main drawback to this imaging strategy is the loss of patient to follow-up [3, 18].

Keeping scanning time as short as possible, briefing the patient on the importance of keeping still during scanning and the use of sedation usually helps prevent or reduce movement artefacts and misregistration errors in ADC map calculation [38].
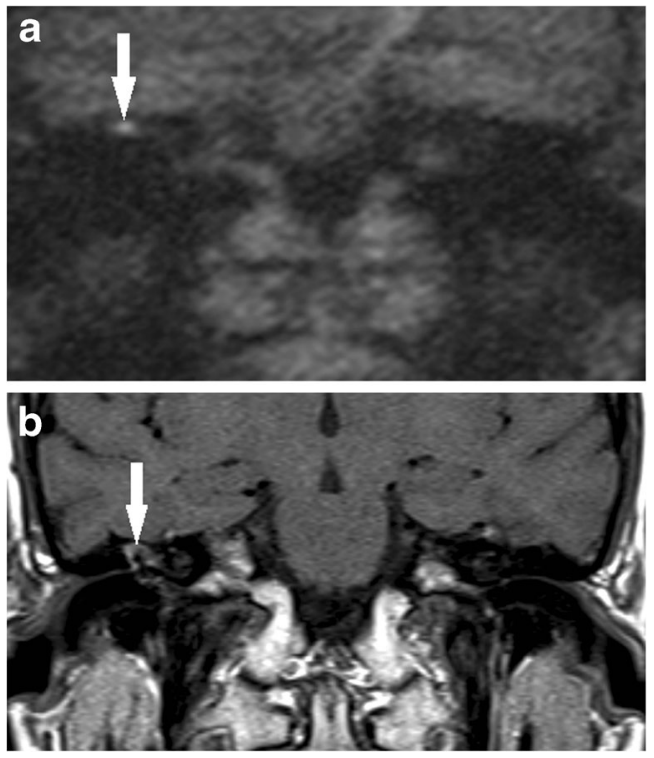

Fig. 10 False positive high DWI signal for cholesteatoma. a The coronal b1000 image shows high signal at the epitympanum, which raised suspicion for residual cholesteatoma. However, b the corresponding non-contrast enhanced coronal T1 weighted image showed high signal (white arrow) and the ADC map intermediate signal and value, both not supporting the diagnosis of cholesteatoma. Surgery confirmed nonspecific inflammation with no cholesteatoma 
Fig. 11 Indeterminate appearance of residual cholesteatoma: Value of ADC. The coronal a T2 weighted and $\mathbf{b}$ b0 DWI images show a small focus of high signal soft tissue at the left hypotympanum, which returns just mildly high signal relative to brain tissue on $\mathbf{c}$ the b1000 DWI image, raising the possibility of a residual pearl (similar to Fig. 9a). d The ADC map demonstrates low signal and value compatible with cholesteatoma, thereby increasing observer confidence. e This was later confirmed as a $3-4 \mathrm{~mm}$ cholesteatoma at the left hypotympanum on second-look surgery
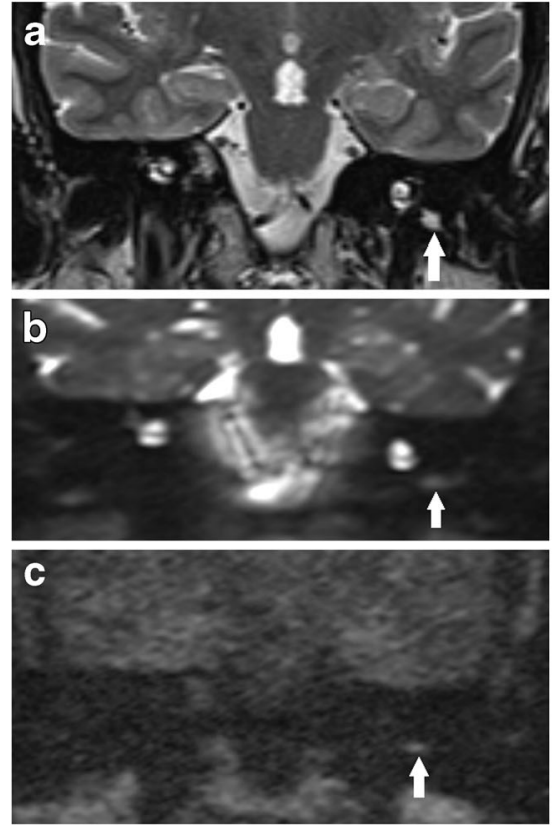

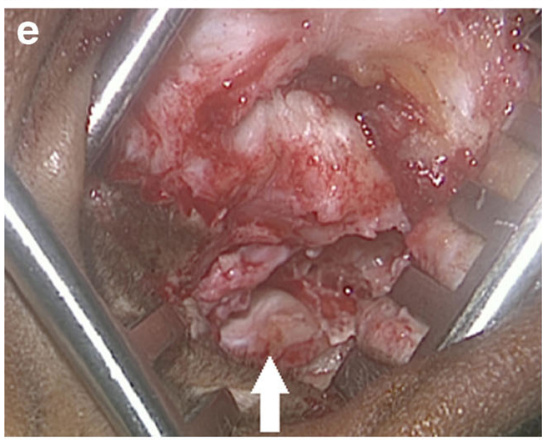

\section{Pitfalls in specificity}

There is a myriad of false positive cases described in the literature for detecting cholesteatoma using non-echoplanar DWI. Commonly in our experience, wax (Fig. 7) or proteinaceous fluid (Fig. 8) /cysts and non-specific inflammation (Figs. 9 and 10) can return high signal on the b1000 images $[3,12,22,23,32,35,38]$. They can also be a result of operative materials such as silastic sheet, bone dust/powder [2, 20, 35], and (calcified) cartilage [38]. There are reports of false positive cases from dental braces artefacts [17], tympanosclerosis [38], cholesterol granuloma [22, 38], and squamous cell carcinoma of the external auditory canal [35].

\section{Navigating past the pitfalls in specificity}

To improve diagnostic performance including specificity, image interpretation should be performed in a clear clinical context and not in isolation. A useful way to resolve disconcordant or questionable DWI high signals is for the radiologists to discuss with the otologists, ideally in a joint clinico-radiological meeting. The treatment history, such as type of operation(s) performed and when, the materials used, surgical confidence of clearance, the extent of initial disease (small pearl in the epitympanum or extensive disease into the mastoid) and current ear status (discharging or quiet), and examination are all relevant and important clinical factors to consider. A small focus of dependant high b1000 signal and restricted diffusion posteriorly at the mastoid is unlikely to represent a cholesteatoma pearl if the initial primary disease was a small epitympanic cholesteatoma which was easily and confidently removed on surgery. Indeterminate DWI signals of low radiological and surgical suspicion for cholesteatoma can easily be followed up with DWI over time to ensure stability or resolution and hence exclude the possibility of cholesteatoma with more certainty (Fig. 8). In addition, by providing feedback to the radiologists following surgery, learning is reinforced and hence better and more confident radiological interpretation is nurtured.

Supplementing qualitative analysis of the DWI images and $\mathrm{ADC}$ map with other MRI sequences and $\mathrm{ADC}$ values can also help improve specificity [12]. Quantitative analysis using ADC
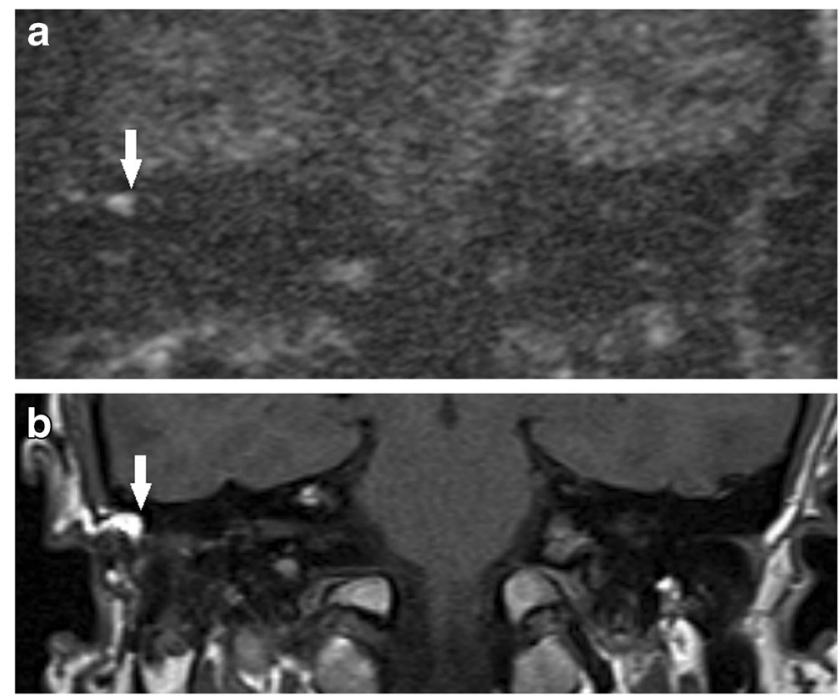

Fig. 12 Value of non-contrast enhanced T1 weighted images. False positive high DWI signal (white arrow) for cholesteatoma was seen on a the b1000 image. b The non-contrast enhanced T1 weighted image showed this (white arrow) to represent subcutaneous tissue just outside the reconstructed post-operative middle ear cleft. Otoscopic examination of the area is normal and following discussion at the clinicoradiological meeting, a follow-up DWI was arranged. This showed stable appearance 
Fig. 13 Using DWI following canal wall down surgery. In a patient who presented with persistent otorrhoea after CWD surgery, a the DWI b1000 images show high signal in the mastoid remnants bilaterally, which corresponded to infected debris/ wax on clinical inspection. b On direct clinical inspection of the left mastoid remnant, which showed the infected debris/wax (white arrow), a bony defect (black arrow) is noted on the medial wall above the bony labyrinth. The defect communicated with extensive residual cholesteatoma hidden deep in the petrous apex, which was demonstrated by high signal soft tissue (white arrow) on the coronal c T2 weighted and d b1000 images
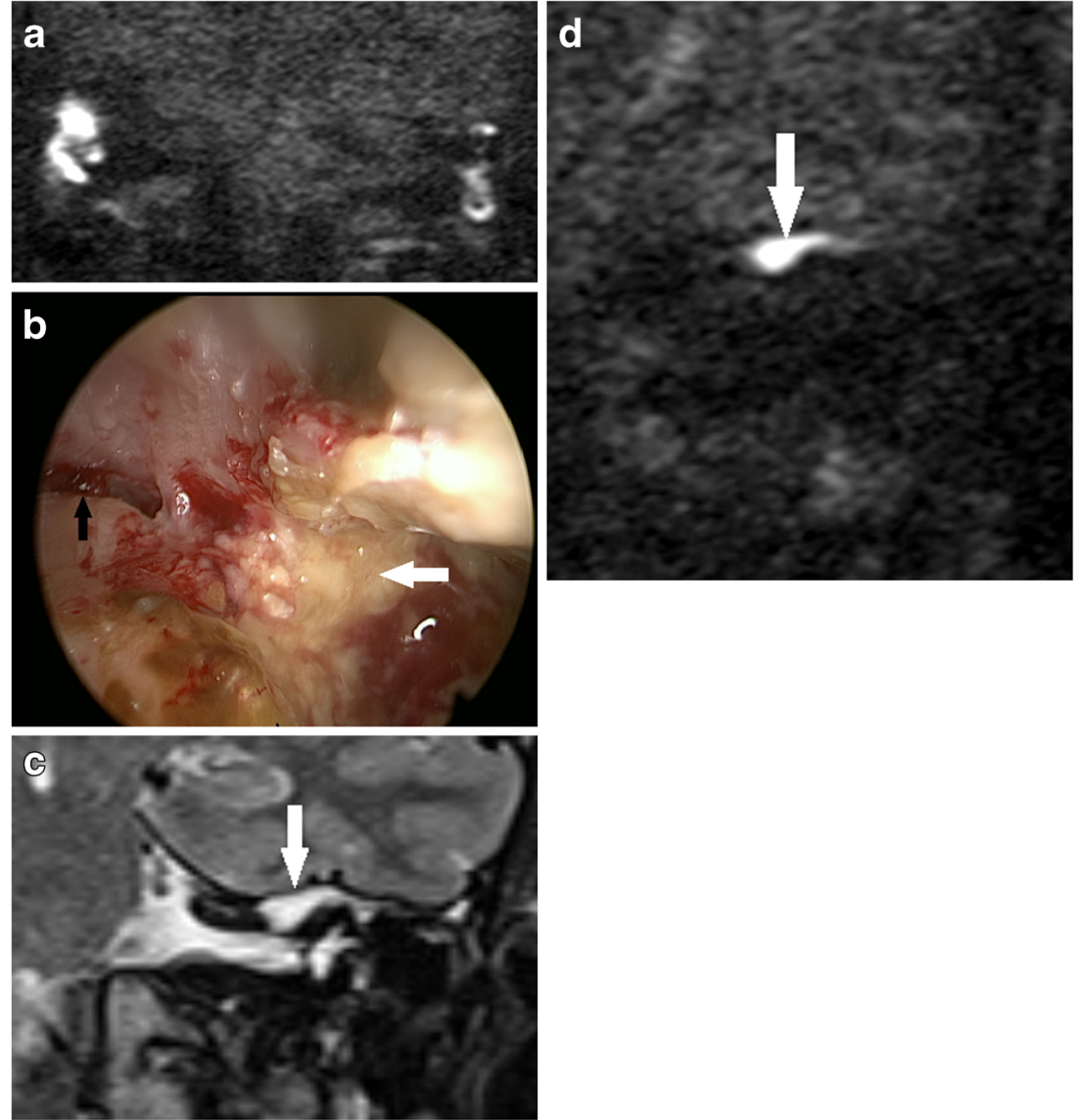

values can provide insight into the nature of the indeterminate lesions because cholesteatoma typically produces significantly lower ADC values than non-cholesteatomatous soft tissue including granulation tissue, proteinaceous fluid or inflammation
[12] (Figs. 9 and 10). Not infrequently, the cholesteatoma pearls formed from residual disease are not large enough to return the characteristic higher signal than brain tissue on the b1000 image and instead return intermediate or borderline high signal
Fig. 14 Using DWI following canal wall down surgery. a DWI b1000 image depicted residual disease (white arrow) at the mastoid tip, also seen as low signal on the $\mathbf{b}$ corresponding non-contrast enhanced $\mathrm{T} 1$ weighted image and a heterogenous, but predominantly high signal on the $\mathbf{c}$ T2 weighted image. d Surgical exploration confirmed residual cholesteatoma pearl (white arrow) walled off in the mastoid tip and was not readily visible on direct inspection at the preceding follow-up clinic. The lesion also had low ADC signal and value
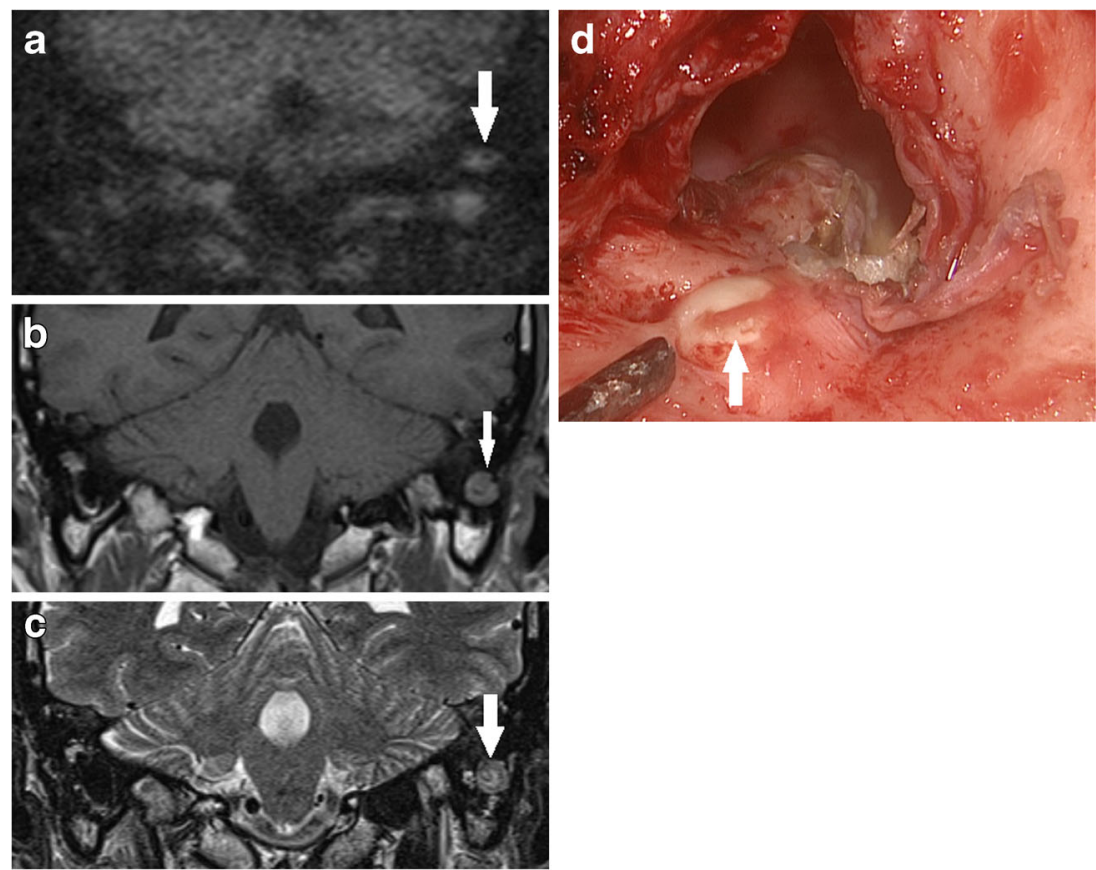
(Fig. 11). They can pose a diagnostic challenge, but corroborating with its $\mathrm{ADC}$ value can help improve the observer's confidence level in detecting cholesteatoma [12]. T1-weighted images may also help in improving specificity of the DWI images, as some proteinaceous fluid or non-cholesteatomatous soft tissue including cholesterol granuloma can return high T1W signal which is not associated with cholesteatoma [4] (Figs. 8, 10, and 12). The location and configuration of the lesion is also informative. Wax in the ear canal can return linear (often parallel) high signal changes on the b1000 images and low signal and values on the ADC map (Fig. 7). As they can depict anatomy better than the DWI images, the T1W and T2W images are especially useful in improving localisation of the lesion and assessing whether spurious DWI high signals mimicking possible cholesteatoma actually lie within or outside the postoperative middle ear cleft (Figs. 7 and 12). Fusion of DWI images with high resolution CT images can depict clearer localisation of DWI signal of post-operative cholesteatoma in relation to the bony anatomy and aid in surgical planning [41], but this would incur additional radiation exposure.

\section{Use of DWI following canal wall down surgery}

The risk of residual or recurrent disease following canal wall down (CWD) mastoidectomy is much lower than that of canal wall up mastoidectomy $[3,5]$. When accumulating keratin occurs within a mastoid cavity, it can often be readily detected and successfully cleared with microsuction in an outpatient setting. In addition, wax has a tendency to accumulate in a CWD mastoid cavity, and this can cause false positive signals on DWI (Fig. 13a). However, there are instances where DWI is especially valuable when disease is not readily visible on otomicroscopic examination, such as disease extension into the petrous apex (Fig. 13) or mastoid tip (Fig. 14), disease deep to an obliterated cavity, in a small reconstructed middle ear cavity or medial to an opaque tympanic membrane reconstruction [42]. Close communication between the radiology and otology teams, with clear clinical information, and a clear indication for imaging is crucial.

\section{Use of DWI in children}

CWU surgery is preferred in children because it obviates the potential requirement for lifelong ear care, improves the fitting of hearing aids when required, and is less likely to lead to otorrhoea associated with swimming [3]. Cholesteatoma in children, however, is more aggressive than adult disease and has a higher rate of recurrent and residual disease postoperatively [43] and the otologist is more inclined to perform mandatory second-look surgery to detect disease. Having said this, a recent large prospective observational study comparing 154 adult cases with 54 paediatric cases in detecting post- operative disease, showed similar good diagnostic performance between the two groups [38]. Hence, clinically, DWI can be considered effective in a paediatric setting as in an adult setting, in which many otologists will have more experience. By avoiding radiation exposure and administration of intravenous contrast medium, DWI is especially attractive for imaging children. However, children are less likely to be tolerant of MRI scanning and may require sedation. In our experience, successful scanning can be achieved without the need for sedation, if the clinician explained the essentials of the procedure to the parent and child beforehand.

\section{Conclusion}

Even though non-echoplanar DWI has a high diagnostic performance in detecting post-operative cholesteatoma, it is not without limitations. The pitfalls of image interpretation need to be recognised and managed appropriately by both radiologists and otologists for it to contribute to effective and safer patient care.

Open Access This article is distributed under the terms of the Creative Commons Attribution 4.0 International License (http:// creativecommons.org/licenses/by/4.0/), which permits unrestricted use, distribution, and reproduction in any medium, provided you give appropriate credit to the original author(s) and the source, provide a link to the Creative Commons license, and indicate if changes were made.

\section{References}

1. De Foer B, Vercruysse J-P, Pilet B, Michiels J, Vertriest R, Pouillon M et al. (2006) Single-shot, Turbo Spin-echo, Diffusion-weighted imaging versus Spin-Echo-planar, diffusion-weighted imaging in the detection of acquired middle ear cholesteatoma. AJNR 27:1480-1482

2. Dubrulle F, Souillard R, Chechin D, Vaneecloo FM, Desaulty A, Vincent C (2006) Diffusion-weighted MR imaging sequence in the detection of postoperative recurrent cholesteatoma. Radiology 238(2):604-610

3. Khemani S, Singh A, Lingam RK, Kalan A (2011) Imaging of postoperative middle ear cholesteatoma. Clin Radiol 66:760-767

4. Más-Estellés F, Mateos-Fernández M, Carrascosa-Bisquert B et al. (2012) Contemporary non-echo-planar diffusion-weighted imaging of middle ear cholesteatomas. Radiographics 32:1197-1213

5. Li PM, Linos E, Gurgel RK et al. (2013) Evaluating the utility of non echo-planar diffusion-weighted imaging in the preoperative evaluation of cholesteatoma: a meta-analysis. Laryngoscope 123:1247-1250

6. Jindal M, Riskalla A, Jiang D, Connor S, O'Connor AF (2011) A systematic review of diffusion-weighted magnetic resonance imaging in the assessment of postoperative cholesteatoma. Otol Neurotol 32: $1243-1249$

7. van Egmond SL, Stegeman I, Grolman W, Aarts MC (2016) A systematic review of non-echo planar diffusion-weighted magnetic resonance imaging for detection of primary and postoperative cholesteatoma. Otolaryngol Head Neck Surg 154(2):233-240

8. De Foer B, Vercruysse JP, Bernaerts A et al. (2010) Middle ear cholesteatoma: non-echo-planar diffusion-weighted MR imaging versus delayed gadolinium-enhanced T1-weighted MR imagingvalue in detection. Radiology 255:866-872 
9. Kuo P, Kanal E, Abu-Alfa A, Cowper SE (2007) Gadolinium-based MR contrast agents and nephrogenic systemic fibrosis. Radiology 242(3):647-649

10. Kanda T, Osawa M, Oba $\mathrm{H}$ et al. (2015) High signal intensity in the dentate nucleus and globus pallidus on unenhanced T1-weighted MR images: association with linear versus macrocyclic gadolinium chelate administration. Radiology 275(3):803-809

11. Le Bihan D, Breton E, Lallemand D, Aubin ML, Vignaud J, LavalJeantet M (1988) Separation of diffusion and perfusion in intravoxel incoherent motion MR imaging. Radiology 168(2):497-505

12. Lingam RK, Khatri P, Hughes J, Singh A (2013) Apparent diffusion coefficients for detection of postoperative middle ear cholesteatoma on non-echo-planar diffusion-weighted images. Radiology 269(2): 504-510

13. Dhepnorrarat RC, Wood B, Rajan GP (2009) Postoperative nonecho-planar diffusion-weighted magnetic resonance imaging changes after cholesteatoma surgery: implications for cholesteatoma screening. Otol Neurotol 30(1):54-58

14. Huins CT, Singh A, Lingam RK, Kalan A (2010) Detecting cholesteatoma with non-Echo Planar (HASTE) diffusionweighted magnetic resonance imaging. Otolaryngol Head Neck Surg 143(1):141-146

15. Rajan GP, Ambett R, Wun L, Dhepnorrarat RC, Kuthubutheen J, Chow Z et al. (2010) Preliminary outcomes of cholesteatoma screening in children using non-echo-planar diffusion-weighted magnetic resonance imaging. Int $\mathrm{J}$ Pediatr Otorhinolaryngol 74(3):297-301

16. Pizzini FB, Barbieri F, Beltramello A, Alessandrini F, Fiorino F (2010) HASTE diffusion-weighted 3-Tesla magnetic resonance imaging in the diagnosis of primary and relapsing cholesteatoma. Otol Neurotol 31(4):596-602

17. Plouin-Gaudon I, Bossard D, Fuchsmann C, Ayari-Khalfallah S, Froehlich P (2010) Diffusion-weighted MR imaging for evaluation of pediatric recurrent cholesteatomas. Int J Pediatr Otorhinolaryngol 74(1):22-26

18. Khemani S, Lingam RK, Kalan A, Singh A (2011) The value of non-echo planar HASTE diffusion-weighted MR imaging in the detection, localisation and prediction of extent of postoperative cholesteatoma. Clin Otolaryngol 36:306-312

19. Profant M, Slavikova K, Kabatova Z, Slezak P, Waczulikova I (2012) Predictive validity of MRI in detecting and following cholesteatoma. Eur Arch Otorhinolaryngol 269:757-765

20. Karandikar A, Loke SC, Goh J, Yeo SB, Tan TY (2015) Evaluation of cholesteatoma: our experience with DW Propeller imaging. Acta Radiol 56(9):1108-1112

21. Lehmann P, Saliou G, Brochart C (2009) 3T MR imaging of postoperative recurrent middle ear cholesteatomas: value of periodically rotated overlapping parallel lines with enhanced reconstruction diffusionweighted MR imaging. AJNR Am J Neuroradiol 30:423-427

22. Suzuki H, Sone M, Yoshida T, Otake H, Kato K, Teranishi M et al. (2014) Numerical assessment of cholesteatoma by signal intensity on non-EP-DWI and ADC maps. Otol Neurotol 35(6):1007-1010

23. Mateos-Fernandez M, Mas-Estellés F, de Paula-Vernetta C, GuzmánCalvete A, Villanueva-Martí R, Morera-Pérez C (2012) The role of diffusion weighted magnetic resonance imaging in cholesteatoma diagnosis and follow-up. Study with the diffusion PROPELLER technique. Acta Otorrinolaringol Esp 63(6):436-442

24. Yamashita K, Yoshiura T, Hiwatashi A et al. (2013) High resolution three dimensional diffusion-weighted imaging of middle ear cholesteatoma at 3.0T MRI: usefulness of 3D turbo field-echo with diffusion-sensitized driven-equilibrium preparation (TFE-DSDE) compared to single-shot echo-planar imaging. Eur J Radiol 82:e471-e475

25. Schwartz KM, Lane JI, Bolster BD Jr, Neff BA (2011) The utility of diffusion-weighted imaging for cholesteatoma evaluation. Am J Neuroradiol 32:430-436
26. Padhani AR, Liu G, Koh DM et al. (2009) Diffusion-weighted magnetic resonance imaging as a cancer biomarker: consensus and recommendations. Neoplasia 11(2):102-125

27. Kim JH, Choi SH, Chung JW (2009) Clinical results of atticoantrotomy with attic reconstruction or attic obliteration for patients with an attic cholesteatoma. Clin Exp Otorhinolaryngol 2(1):39-43

28. Blanco P, González F, Holguín J, Guerra C (2014) Surgical management of middle ear cholesteatoma and reconstruction at the same time. Colomb Med 45(3):127-131

29. Lecler A, Lenoir M, Peron J, Denoyelle F, Garabedian EN, Pointe HD et al (2015) Magnetic resonance imaging at one year for detection of postoperative residual cholesteatoma in children: is it too early? Int J Pediatr Otorhinolaryngol 79(8):1268-1274

30. Akkari M, Gabrillargues J, Saroul N, Pereira B, Russier M, Mom T et al. (2014) Contribution of magnetic resonance imaging to the diagnosis of middle ear cholesteatoma: analysis of a series of 97 cases. Eur Ann Otorhinolaryngol Head Neck Dis 131(3):153-158

31. Sharifian H, Taheri E, Borghei P, Shakiba M, Jalali AH, Roshanfekr $\mathrm{M}$ et al. (2012) Diagnostic accuracy of non-echo-planar diffusionweighted MRI versus other MRI sequences in cholesteatoma. J Med Imaging Radiat Oncol 56(4):398-408

32. von Kalle T, Amrhein P, Koitschev A (2015) Non-echoplanar diffusion-weighted MRI in children and adolescents with cholesteatoma: reliability and pitfalls in comparison to middle ear surgery. Pediatr Radiol 45(7):1031-1038

33. Ilıca AT, Hıdır Y, Bulakbașı N et al. (2012) HASTE diffusionweighted MRI for the reliable detection of cholesteatoma. Diagn Interv Radiol 18:153-158

34. Elefante A, Cavaliere M, Russo C, Caliendo G, Marseglia M, Cicala D, et. al. (2015) Diffusion weighted MR imaging of primary and recurrent middle ear cholesteatoma: an assessment by readers with different expertise. BioMed Res Int 597896

35. Garrido L, Cenjor C, Montoya J, Alonso A, Granell J, GutierrezFonseca R (2015) Diagnostic capacity of non-echo planar diffusion-weighted MRI in the detection of primary and recurrent cholesteatoma. Acta Otorrinolaringol Esp 66(4):199-204

36. Nagai N, Tono T, Matsuda K, Toyama K, Kawano H, Kodama T (2008) Value of non echo-planar diffusion-weighted magnetic resonance imaging in the detection of middle ear cholesteatomea. Mediterr J Otol 197-202

37. Vaid S, Kamble Y, Vaid N et al. (2013) Role of magnetic resonance imaging in cholesteatoma: the Indian experience. Indian J Otolaryngol Head Neck Surg 65(suppl 3):485-492

38. Nash R, Wong PY, Kalan A, Lingam RK, Singh A (2015) Comparing diffusion weighted MRI in the detection of postoperative middle ear cholesteatomain children and adults. Int $\mathrm{J}$ Pediatr Otorhinolaryngol 79(12):2281-2285

39. El Mogy SA, Mazroa JA, El Ghaffar MA, El Mogy MS, El Mogy IS (2011) Evaluation of acquired cholesteatoma with PROPELLER diffusion imaging. Egypt J Radiol Nucl Med 42:9-17

40. Steens S, Venderink W, Kunst D, Meijer A, Mylanus E (2016) Repeated postoperative follow-up diffusion-weighted magnetic resonance imaging to detect residual or recurrent cholesteatoma. Otol Neurotol 37:356-361

41. Plouin-Gaudon I, Bossard D, Ayari-Khalfallah S, Froehlich P (2010) Fusion of MRIs and CT scans for surgical treatment of cholesteatoma of the middle ear in children. Arch Otolaryngol Head Neck Surg 136(9):878-883

42. Nash R, Kalan A, Lingam RK, Singh A (2016) The role of diffusion-weighted magnetic resonance imaging in assessing residual/recurrent cholesteatoma after canal wall down mastoidectomy. Clin Otolaryngol 41(3):307-309

43. Nevoux J, Lenoir M, Roger G, Denoyelle F, Ducou Le Pointe H, Garabédian EN (2010) Childhood cholesteatoma. Eur Ann Otorhinolaryngol Head Neck Dis 127:143-150 